Waves 8 Oscillations in the Solar Atmosphere:

Heating and Magneto-Seismology

Proceedings IAU Symposium No. 247, 2007

R. Erdélyi \& C. A. Mendoza-Briceño, eds.

(C) 2008 International Astronomical Union doi:10.1017/S1743921308014804

\title{
A search for oscillating loops in Solar-B XRT observations
}

\author{
Antonia Savcheva ${ }^{1}$ and Edward DeLuca ${ }^{1}$ \\ ${ }^{1}$ Harvard-Smithsonian Center for Astrophysics, 60 Garden st, Cambridge, MA02139, USA \\ email: asavcheva@cfa.harvard.edu and edeluca@cfa.harvard.edu
}

\begin{abstract}
Between November 2006 and March 2007, on several occasions, XRT has been taking high-cadence one or two filter observations of prominent active regions on the disk. We took these datasets and conducted a quick search for acoustic brightness oscillation in loops. We concentrated our search on flaring active regions. Here we present the preliminary results of this search. We found one active region - NOAA 10953 from 27 April - 02 May 2007 that had indications of acoustic oscillations with periods around $5 \mathrm{~min}$, as well as multiples of this period, and one 40 min period that we associate with periodic heating of the loops. An interesting result is that all the loops in the active region seemed to oscillate with the same set of periods, only the power in the FFT was different and maybe dependent on the magnetic field strength.
\end{abstract}

\section{Introduction}

The search and modeling of acoustic oscillation in different structures on the Sun had been ongoing for about 15 years. Such searches have been conducted at all available wavelengths that space- and ground-based instruments can observe and hence at different heights in the solar atmosphere. The only underused wavelengths is the X-rays. In this paper we present an attempt for a search of acoustic oscillations in data acquired with the X-ray Telescope (XRT) on Hinode. We discuss the observation datasets, the search methods, and finally, the results for AR 10953, which revealed the existence of oscillations as well as the periodic heating of the loops in the region.

\section{Observations}

On multiple days between November 2006 and May 2007 XRT has been gathering high-cadence observations of active regions in different filters in order to study the dynamics of different oscillatory and eruptive phenomena. In the search for oscillating loops we considered only observations with highest available cadence - 20-40s. We also concentrated on observations taken around the time of increased X-ray flux in the GOES data. This left us with the observations conducted on February 12, March 1, April 27, April 30, and May 02, 2007. Most of the observations were taken continuously in the Al_poly filter. Some of the data was in the Ti_poly and Al_mesh filters. Unfortunately, for the sake of high cadence a sequence of data has to be in one filter, which makes a multi-filter temperature analysis impossible.

\subsection{The Search for Oscillations and Resuts}

Initially, all data was cleaned from cosmic ray particle hits, normalized by exposure time, and processed for instrumental noise. The datasets were divided into a number of 6 -hour subsets because computational memory requirements. Then three types of search 
Table 1. Values for the period of all significant pekas in the FFT. All periods are present in all considered loops.

\begin{tabular}{lccc}
\hline Period [min] & Significance & Possible interpretation & Persists to later datasets \\
\hline 4.21 & $>3 \sigma$ & 5 min oscillations & no \\
5.74 & $>3 \sigma$ & 5 min oscillations & no \\
8.81 & $3 \sigma$ & & no \\
11.68 & $>3 \sigma$ & $2 \times 5.74$ min & yes \\
17.43 & $3 \sigma$ & $3 \times 5.74$ min & yes \\
40.22 & $3 \sigma$ & Periodic heating of the loops & no \\
\hline
\end{tabular}

techniques ware performed on all images to look for oscillations in the brightness of X-ray loops in the active regions. First, an animated image sequence and a running difference movie were constructed and inspected by eye for obvious signs of oscillations. Several loops were identified in the active regions and the light curves of several points along the loops were obtained. The light curves were also inspected for apparent periodicities. Fast Furrier Transform (FFT) was performed on the light curves of several points from the loops and the significance of detected peaks was determined.

For most of the data we processed we did not find any apparent oscillations in the running difference movies or the light curves and the peaks in the FFT did not have more than $2 \sigma$ significance. The only active region where we detected oscillations visually and on the FFT was AR 10953. Luckily, we had three days of data on this active region - 27 and 30 April and 02 May. The GOES data for the 27-th showed a number of B-class flares, so it was a suitable date to concentrate or analysis.

The inspection of the movies and light curves for these dates was not conclusive of any oscillations. However, the movies indicated possible periodicity of heating the loops with a period of about $50 \mathrm{~min}$. The FFT revealed that a number of periods (see Table 1) proved to be highly significant. These periods appear in the FFT of all the loops in the active region with different power (see Fig.1), which an interesting result. Other points of the image, outside the active region, were checked for oscillations to rull out the possibility of these oscillations being intrinsic to the whole images. It was confirmed that the detected oscillations indeed belong to the loops. We also found that two of the periods persisted on all days of the observation.

The Photospheric B-field strength from chromospheric magnetogram was extrapolated to the corona and the strength of the field was determined for the separate loops. Possible correlation between the power in the prominent peaks and the magnetic field strength in the loops has been noticed. The magnetic field strength had three different values in the different loops - 10G, 80G, 200-300G. The power of the peaks in the power spectrum is greater with increasing B-field strength.

As mentioned, inspection of the animated sequence of images from 27 April and the running difference, showed that a periodic heating of the whole loop structure was taking place. By eye we determined a period of about $50 \mathrm{~min}$ and the FFT showed a strong peak at $40 \mathrm{~min}$. This is mostly visible in loops 1 and 3 as marked on Fig. 1. The consecutive images show how loop 1 brightens and fades with time and the heating starts at the footpoint and propagates along the loop. We determined the velocity of the heating front to be in the range $500-700 \mathrm{~km} / \mathrm{s}$ for the different events. For comparison, for $\mathrm{B}=10 \mathrm{G}$ and assumed number density in the loop of $10^{9} \mathrm{~cm}^{-3}$, the Alfven speed is $690 \mathrm{~km} / \mathrm{s}$.

Similar activity was observed on 30 April and 02 May, although not as periodic. The TRACE data from the same time also showed significant dynamics at the places associated with loops 1 and 3 . 

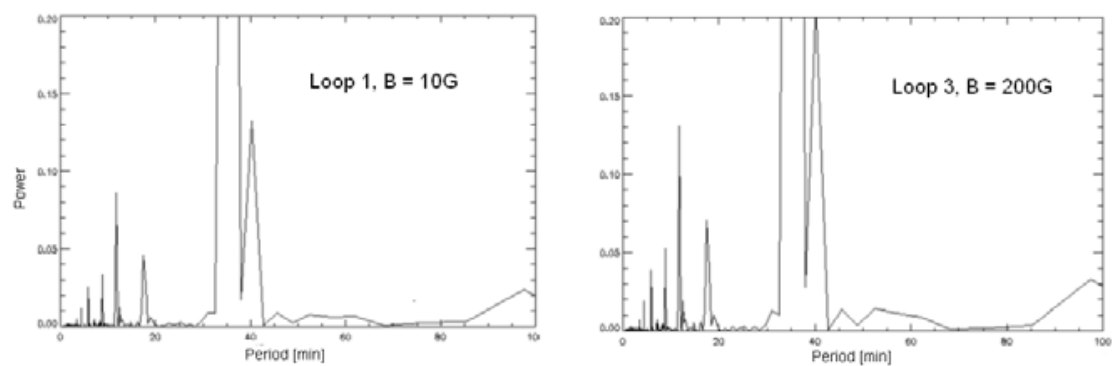

Figure 1. Power spectra of the lightcurve at fixed points in two loops - Loop 1 (left) and Loop 3 (right). All higher peaks except the terminated one in the middle ( periodic gaps in the data) are intrinsic.
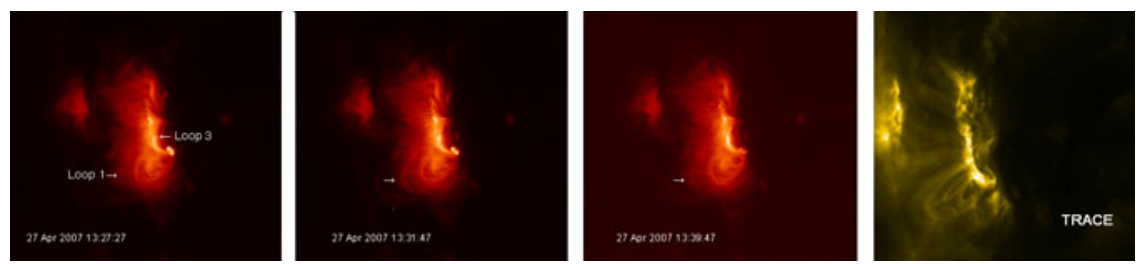

Figure 2. Different instances of time from the April 27 dataset. The loops that we used for our analysis are marked on the leftmost image. Notice how Loop 1 changes brightness in consecutive images. The rightmost image is a TRACE image from the same time of the same active region.

\section{Conclusions}

We presented some preliminary results from the search for oscillations we performed on XRT data. We found several strong oscillation periods in the loops of AR 10953. We associated some of them with the $5 \mathrm{~min}$ oscillations and one with periodic heating. We think that a correlation between the magnetic field strength and the power in these frequencies is probable. An interesting result is that $90 \%$ all the loops in the active region present the same oscillations. We will continue to look for similar behavior in more XRT data.

\section{Acknowledgements}

Hinode is a Japanese mission developed and launched by ISAS/JAXA, collaborating with NAOJ as a domestic partner, NASA and STFC (UK) as international partners. Scientific operation of the Hinode mission is conducted by the Hinode science team organized at ISAS/JAXA. This team mainly consists of scientists from institutes in the partner countries. Support for the post-launch operation is provided by JAXA and NAOJ (Japan), STFC (U.K.), NASA, ESA, and NSC (Norway). 